\title{
Right robotic-assisted thoracoscopic lower lobectomy
}

\author{
Sasha Stamenkovic ${ }^{1}$, Franca Melfi ${ }^{2}$ \\ ${ }^{1}$ The Thorax Centre, St Bartholomew's Hospital, London, UK; ${ }^{2}$ Robotic Multispecialty Centre for Surgery, Minimally Invasive and Robotic \\ Thoracic Surgery, University Hospital of Pisa, Pisa, Italy \\ Correspondence to: Sasha Stamenkovic. The Thorax Centre, St Bartholomew’s Hospital, London, UK. Email: sasstamenkovic@btinternet.com.
}

\begin{abstract}
The right lower lobectomy is considered one of the easier resection operations performed by an open technique. The posterior approach for the video-assisted thoracoscopic surgery (VATS) version of this is similar, as instruments and staplers pass up the fissure. When moving to the robotic platform with the camera and instruments entering from caudal ports, the equal view of the lobe, fissure and both hila allow for a more balanced operation. This article aims to discuss the technology of robotic-assisted surgery and the optimal surgical techniques to enable precise and safe resection of the lower lobe.
\end{abstract}

Keywords: Robot-assisted; thoracoscopic; lobectomy

Submitted Nov 22, 2018. Accepted for publication Jan 25, 2019.

doi: $10.21037 /$ acs.2019.03.04

View this article at: http://dx.doi.org/10.21037/acs.2019.03.04

\section{Introduction}

We will discuss using the technology of the surgical robot to its best advantage followed by the steps to perform the operation. As there is predominantly one solid-organ robot in use, the discussion will relate to this. Our understanding has come a long way since the first explanation of this technology for the chest by Melfi and her team in Pisa (1), as well as Park and colleagues in Memorial Sloan-Kettering (2). If we accept the fact that the robot allows better vision and complete articulation of effector arms (Figure 1), then it follows that anything that is done or omitted by the surgeon that worsens operative vision warrants discussion. The lobectomy procedure described herein is an advanced robotic operation that brings together many different operative techniques.

\section{Technology}

\section{Camera}

The superior vision of the robot camera plays a large part in the success of a robotic operation. However, the immersive biscopic 3DHD vision of which the console surgeon has the benefit, and the first assistant's planar 2D view are very different. This needs to be taken into consideration by the console surgeon when the first assistant's tasks are to be carried out, such as by increased clearing of the visual field and zooming in. The camera should always be pointing to the centre of the field. If not, an inferior view, poor visual resolution, fluctuating light intensity and poor depth of vision results. White swabs will substantially decrease light intensity as will dark swabs and blood so it is imperative to remove these. The camera should not be operating in the shade of other instruments, anatomical structures or the port end.

The camera should point horizontally with a neutral horizon to prevent disorientation of the surgeon or first assistant. Intentional rotation may temporarily be required to operate or to help find instruments. Different angulation facilitates visualization of different structures at different stages of the operation (Figure 2). Thirty degrees up (30 up) allows visualization of the chest wall, port placement, instrument insertion and removal as well as dissection of the chest wall. Thirty degrees down (30 down) enables vision of the lung, hila and soft tissues. Zooming in with 30 up results in a concave visual arc so the digital zoom may be better. Software options can swap hands when looking caudally such as when dissecting the lung off the diaphragm. Care should be taken to avoid undue impact on the patient. Thirty down may be optimal for the surgical view but can result in a more horizontal arm causing direct pressure or levering in the intercostal space.

The camera should not contact other instruments, anatomical structures or swabs, as time will be lost cleaning 


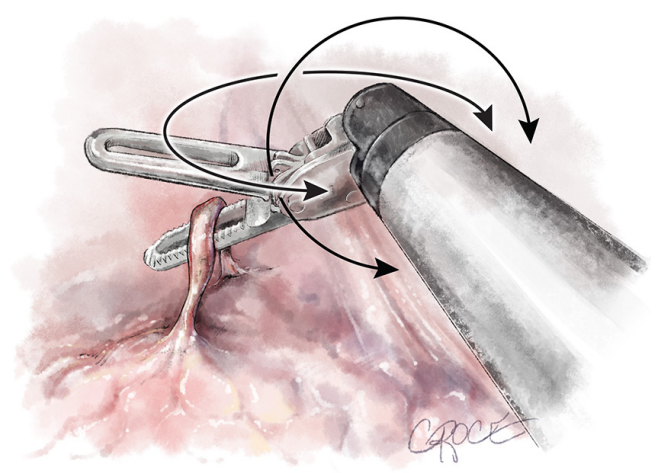

Figure 1 The robotic retractor has two spindles orientated at 90 degrees to each other, which together with the rotation of the shaft provides full 360 degree rotation and access.

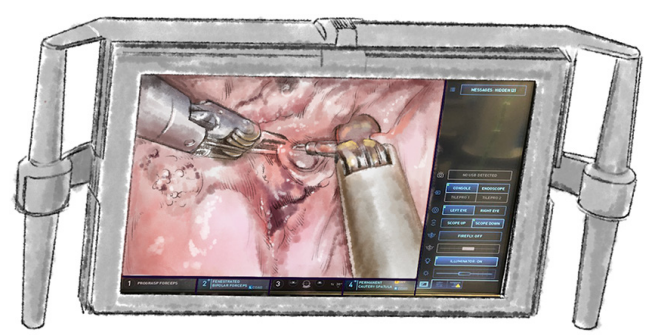

Figure 2 A normal view for the first assistant, with information showing the instruments in use, the horizon angle of the camera, the view angle of the camera (0 degrees or 30 up/down).

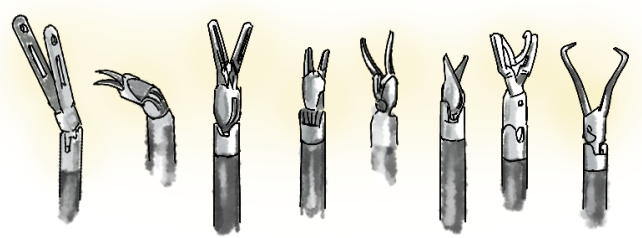

Figure 3 The various ends of the robotic instruments that can be utilised.

the camera. Diathermy smoke or debris will also obscure the camera if too close. If indocyanine green is used then fluoroscopic vision is achieved with switching to Firefly ${ }^{\circledR}$.

\section{End arm effectors (Figure 3)}

The surgeon can use many different instruments with specific characteristics. The retractors provide differing tissue grasping force and tissue-handling. Some surgeons use swabs or patties to retract lung tissue, others grasp the lung directly. The instruments have different degrees of pointedness, so they should be evaluated during simulation to determine optimal use and potential dangers.

Some have short hinges readily maneuverable around structures, whereas others have a longer hinge e.g., staplers and energy sealers/cutters, requiring care when moving from one to another. The port may be partially extracted to allow tension-free use of these. The team should register how many times each respective instrument has been used as well as its condition.

Monopolar or bipolar diathermy can be used, both with advantages and disadvantages. Monopolar allows quick dissection but generates thermal spread which might cause damage to adjacent structures. Dissecting away from vascular structures is safer. Bipolar instruments permit dissection around important structures such as the phrenic nerve and pulmonary artery sheath, however the dissection is slower and the jaws need to be slightly open to grasp and cauterize tissue.

Clips and endowristed staplers come in different lengths and compressions, so tissues have to be approached in different ways to ensure appropriate and safe closure.

The robotic suction/irrigator has a soft fully articulated end with sufficient suction to evacuate large clots, more powerful than an anaesthetic bronchial suction tube which is sometimes used. The irrigation and suction are both foot-operated, which facilitates switching between the two for precise operating with optimal visibility. Non-robotic suction/irrigation devices are not controlled by the console surgeon, are non-articulating with a sharper end that can potentially cause damage and may be too short requiring the first assistant to exert extra pressure to reach the target area. When the surgeon wants to clear around pulmonary arteries and veins, as well as bronchi, there will undoubtedly be lymph nodes or small vessels present, both of which bleed with very little pressure. The robot device allows very precise manipulation, mobilisation and suction of these tissues and may also be carefully advanced while suctioning and irrigating. The angle of approach of each instrument has to be planned thoroughly. In the latest editions of the robot, there is the possibility of 'camera hopping' as well as moving an instrument into any of the ports. This allows many different instrument-camera combinations and views and thus enhances the ability of the robot to move around corners or awkward positions. However, each time that the camera is hopped to another port, the view has changed, requiring a moment to re-orientate. The surgical team should practice the 'pit stops' of changing instruments around and cleaning the camera so that operative efficiency is maximized. 


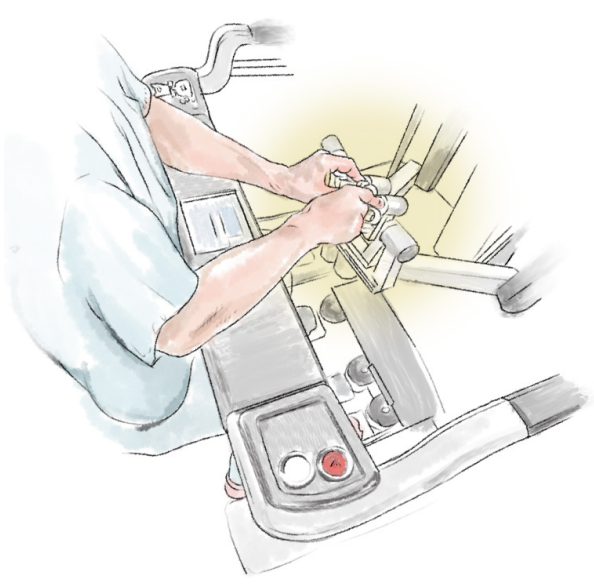

Figure 4 An understanding of the potential conflicts are key to safe and precise operating.

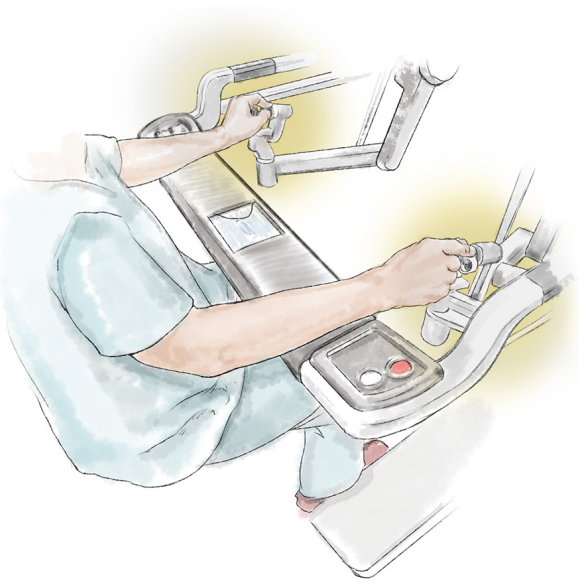

Figure 5 The outer parts of the console.

\section{Advantages of operating with a surgical robot}

There are four arms to a modern robot with the ability to interchange the camera and the instruments. The 4th arm is most useful when it has an instrument retracting tissue sufficiently to allow the other two arms to operate closely together, eliminating the difficulty of one-handed operation or pushing as required in most standard video-assisted thoracoscopic operations. Picking up the tissue with one hand and cutting or dissecting it, with or without energy, is necessary in robotic surgery as in open surgery. It represents a totally endoscopic procedure through tiny holes with careful dissection on one side and counter-traction on the other, while the larger piece of the organ or tissue is optimally retracted at a distance.

In a totally endoscopic robotic-assisted operation carbon dioxide is used to increase lung deflation, diathermy-fume dispersion and pushing down the hemi-diaphragm, permitting sub xiphoid access (3) for insertion of instruments or specimen extraction. With the advent of single port technology, this approach will be employed more and more. A maximum pressure $(<8 \mathrm{mmHg}$ ) needs monitoring especially with gas-bottle exchange as some machines default to a much higher pressure. Pre-operative dehydration protocols can cause right ventricular compression. If the $\mathrm{CO}_{2}$ line is kinked or the gas leaks or runs out the anatomy will move, so instruments should be moved to the chest wall to prevent inadvertent damage to structures.

Instruments may need to be exchanged from one hand to the other when at the extremes of the chest as the limits of movement are reached. Articulation of the tip can be changed while holding tissue exerts no extra pressure and this helps to reduce shade, improve vision or overcome internal conflicts. Self-retracting with the shaft and elbow is possible whilst still operating with the tip.

\section{Haptic feedback}

The superior 3DHD visual experience overcomes the need for tactile feedback providing more visual cues regarding the different densities of tissues. As the learning curve progresses, the visual cortex seems to take over from the parietal cortex. When the instrument is run over the interface of hard to soft tissue then blocking, deformation and blanching is seen; when mobilizing an entrance and exit, pulsation occurs as a vessel is freed. All these visual cues enable careful instrument use. Visual proprioception is achieved with the left and right hands closely approximated.

\section{Instrument conflicts (Figures 4-9)}

These include the following six: the master controllers clashing against themselves or the console; the external arm; the internal arm close to the invisible port mistakenly moving ipsilateral arms together; the visible tip contact potentially fracturing the wrist/cable mechanism; and limiting the robot arm by direct pressure on the patient.

\section{Communication}

Patient safety is dependent on excellent communication between the console surgeon and the first assistant with use of unambiguous words. The first assistant should clearly state what their intended action is and it must be backed up by a confirmatory statement from the console surgeon, before 


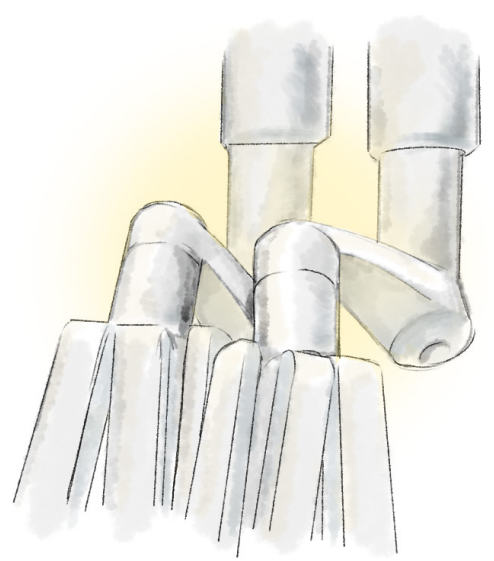

Figure 6 Conflicts can occur if the large arms/joints interfere with normal movement.

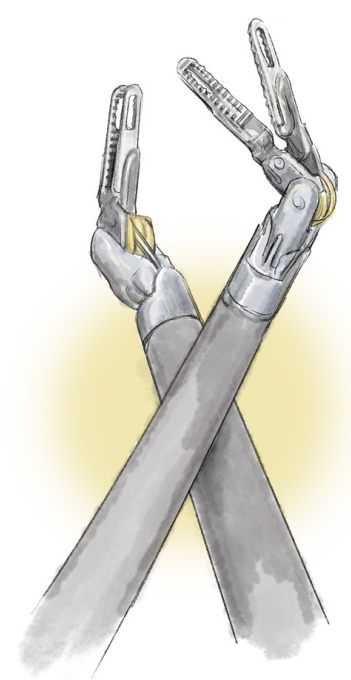

Figure 7 The arms must be kept side-by-side to avoid crossing each other and obstructing movement.

the action is performed. There should be a system of dryrun emergency scenario rehearsals in place before operating as a team, to ensure effective responses to significant airway problems and bleeding issues if they arise (Table 1).

\section{Operation}

\section{Team considerations}

All scrub, anaesthetic and surgical team members must be trained in robotic-assisted operations, and understand the parts, language and sequence of events when converting to a robotic approach.

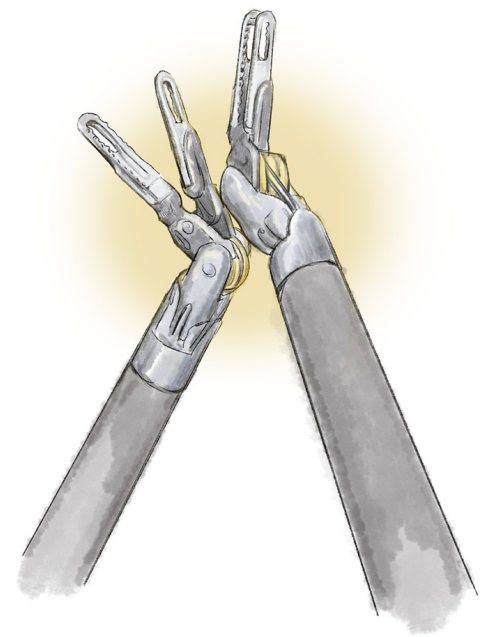

Figure 8 Care must be taken to avoid touching the ends of the instruments. This can lead to inefficient movements as well as instrument damage.

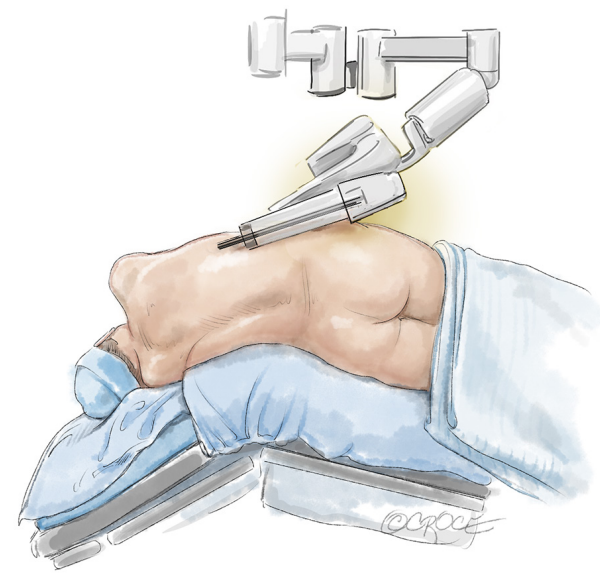

Figure 9 The movement of the robotic arms can also be limited by the positioning of the patient and the patient's hip or shoulder.

\section{Patient selection}

Although robotic operations should be considered for all patients, there may be some limitations relating to the appropriate patient cohort, especially when learning, to permit a team to progress steadily. Challenges may arise for patients with either too low or too high a body mass index [note that Cerfolio has tabulated the ideal patient body habitus (4)], those with poor respiratory reserve or significantly impaired ventricular function. The tumour should be located peripherally with easy hilar and mediastinal access and no obvious extra-capsular or calcified 


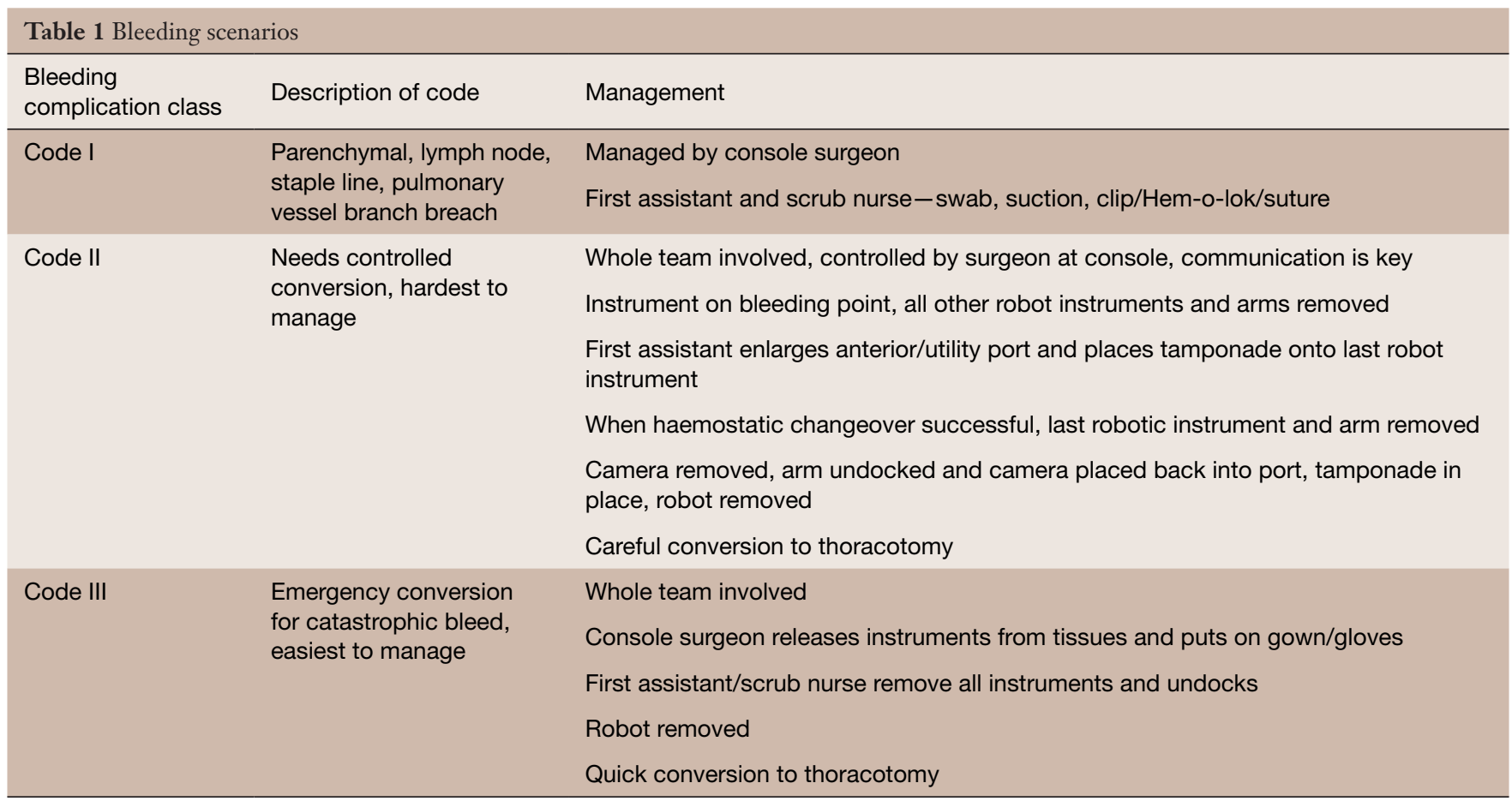

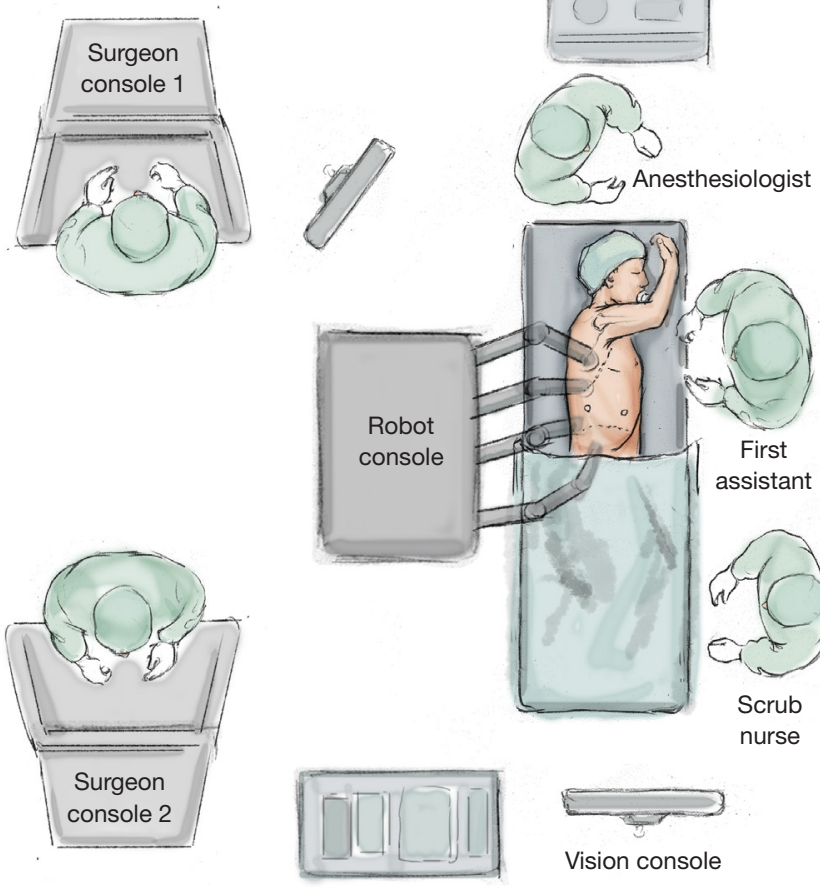

Figure 10 Traditional operating room set-up. lymph nodes, with vessels and bronchi easily encircled. Predictive planning with $3 \mathrm{D}$ reconstruction is useful. Particular care should be exercised in cases of significant kyphosis as the heart is much more anterior in these cases.

\section{Preparation}

Adjusting the operating table (Figure 10) helps to prevent pressure of the ports, port side-arms, robot arms or instruments on the patient, and also decreases the limitation of the robot-arm movement (Figure 11). If a conflict occurs, the carbon-dioxide supply may be disrupted. The use and position of arm holders, vacuum bean-bags and other stability devices must be in relation to the operating position of the robot and its arms.

\section{Port placement, docking of the system and insertion of instruments}

Each system has a prescribed positioning and port-to-port distance to prevent conflicts. Earlier systems needed a more U-shaped port configuration due to the wider profile of the arms and the splayed-out joints of the system. Different views will be obtained from 0 - and 30-degree scopes with some surgeons changing port position to improve view and to 


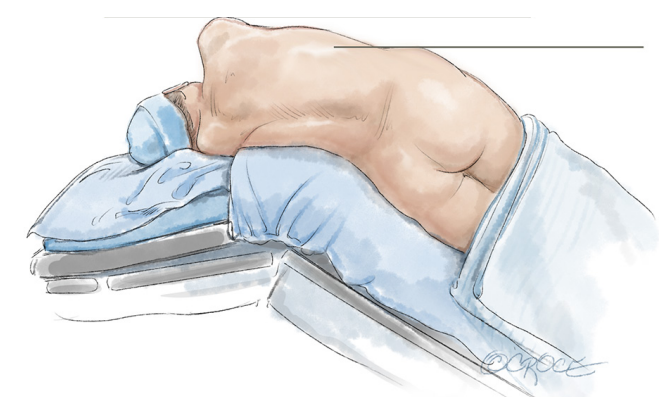

Figure 11 The position of the patient should have the hip lower than the shoulder to prevent the hip from interfering with the robotic arm movement.

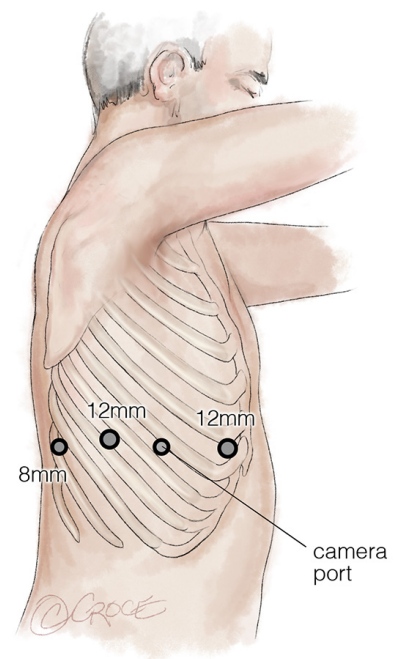

Figure 12 There are several suggested port sites available to facilitate robotic operation.

operate on different lobes. Higher interspaces may be chosen for right upper lobectomies to provide a better view of the trunk and artery/bronchus interface superior to the lung. In a totally endoscopic operation, it is imperative to keep the incision to the defined diameter of the port, to prevent carbon dioxide loss and the non-trochared cannula is used to imprint the skin to guide the size of this incision. A needle may be used to enter the chest under direct vision to accurately incise and place ports (Figure 12). Once the cannulae are in the rib spaces and $\mathrm{CO}_{2}$ is insufflated, they are adjusted to the marked 'remote centre', which includes a fulcrum that permits full rotation of the robotic arm and port cannula with minimal rib-space levering pressure. The robot is then brought towards the operating table and positioned appropriately for its system type. The later versions of the robot have laser guidance for this purpose and once in place, camera targetfollowing allows correct orientation of the arms in. Each arm is yoked or 'docked' with its cannula, and the camera is used to ensure that each still has the remote centre optimally placed. The instruments are chosen dependent on what the first task is and placed under direct vision. A low intercostal, sub-xiphoid or subcostal $12 \mathrm{~mm}$ assistant port can be made as long as there is sufficient room between the pericardium and the diaphragm, to insert instruments and swabs, retrieve lymph nodes and pass the retrieval bag into and out of the chest with the specimen. The use of a non-intercostal port can allow retrieval of significantly air-trapped lobes and, in the authors' experience, tumours up to $15 \mathrm{~cm}$.

\section{Resection}

As a general rule, the easiest part of the operation should be performed first to allow the console surgeon to adapt to the visual field and to develop a feel for the instruments and tissues. Adhesiolysis is done first and in severe cases, the camera port can sweep away immediately visible adhesions, with the benefit of carbon dioxide, until another port can be placed. A one-handed adhesiolysis can continue until a further port is placed. Once all ports are in position, the robot can then be brought in and docking can occur. The division of the inferior ligament and dissection of the level 9 lymph node is then carried out. This is achieved by lifting the lower lobe in a cranial direction with right and left instruments, with or without swabs. Once the lobe is in just the right position to prevent any overhanging shelf or shadow, the 4th arm, which is the most peripheral of the two same-sided arms, is used to hold this in optimal retraction. The boundaries of the inferior vein are then explored using bimanual manipulation, identifying the middle lobe vein. As further division of the ligament and tissues around the vein proceeds, then a corresponding amount of increased retraction is required with the fourth arm. Careful hilar dissection anteriorly and posteriorly may expose other lymph nodes including the subcarinal station, which can be removed. The interface between the vein and the next structure is identified and the vein is mobilised to reveal an entrance and an exit before the Cadiere is used to move around it and a sling is placed.

The lobe is allowed to fall back into its neutral anatomical position by releasing grip of the retracting instruments and, after patting the lobe down, the fissure is explored. If the grading of the fissure (5) is favourable, the sheath of the lower lobe artery is searched for. It is best to stay on the lateral and medial margins of the artery rather than bearing down on 
it, to prevent injury. If the artery is too difficult to mobilize , even with the visual cues of pulsation and difference in colour present, then the resection turns to a fissure-last technique with either a posterior-anterior approach or more commonly an inferior-superior approach. Recognition of the anatomy is critically important if this is the case as the structures are not in the anatomical position and care must be taken to prevent inadvertent division of an artery, vein or bronchus.

If the technique is anterograde then the artery sheath is breached and $1 \mathrm{~cm}$ of tissue either side is cleared to make way for the Cadiere to go around in preparation for the larger stapler, bluntly dissecting in one direction and then at 90 degrees to it (90-90, fig) before a sling is placed. The best port is chosen to allow an endowristed or external stapler to be placed with the most ease, the least angulation and equally the least pressure on adjacent structures. The stapler is placed with no tension on the sling, and gently held in position by the console surgeon before removal of the sling and division. After this the lobe is rolled and held back up to its optimal position as before and the inferior vein divided in the same way. It is sensible when dividing vascular structures to know where the nearest swab is and to have a clamping instrument (Cadiere fenestrated, curved bipolar forceps) proximal to the stapler in case of stapler misfire.

If a retrograde technique is employed then the vein is divided with an endowristed or external stapler. The bronchus is subsequently exposed, mobilized and divided, followed by the artery. The remaining lymph nodes are exposed and removed with careful haemostasis.

\section{Completion}

The final division of the fissure is the last part of the operation, and the separated lobe is placed in that part of the chest that will allow easy extraction, along with all swabs and slings. The lobe is removed through the assistant port in a $550 \mathrm{~mL}$ Espiner sac. At this point, it is likely that there will be loss of $\mathrm{CO}_{2}$. Therefore, the console surgeon should expect the chest anatomy to contract and so pre-emptively move the instruments into positions of safety.

Paravertebral or intercostal blocks are positioned under camera vision. Insufflation of the airway may have already been performed before separation of the lobar bronchus. Testing the stump at the end of the procedure has a potential risk of barotrauma or peripheral showering of the parenchyma so may not be performed. The instruments are removed under direct vision, and the camera is replaced into a port, undocked. Irrigation ensues and at this time the table may be moved into a steep Trendelenburg position to facilitate suction of all the fluid. Once the table is righted, a drain is placed and the lung is allowed to inflate under direct vision with some degree of positive pressure, before the camera is finally removed and the port sites closed in layers.

A successful robotic program requires all members of the team, not just the theatre staff, to have been exposed to the robotic platform in operation. Only then will the wider team understand the purpose of using this technology and adjust their care and treatment of the patient accordingly. This will enable optimization of the early postoperative care of the patient from all members of the multidisciplinary thoracic team to facilitate a safe and rapid recovery.

\section{Acknowledgements}

None.

\section{Footnote}

Conflicts of Interest: S Stamenkovic is a proctor for Intuitive Surgical and has held contracts for advisory boards with Medtronic and Ethicon. F Melfi has no conflicts of interest to declare.

\section{References}

1. Melfi FM, Menconi GF, Mariani AM, et al. Early experience with robotic technology for thoracoscopic surgery. Eur J Cardiothorac Surg 2002;21:864-8.

2. Park BJ, Flores RM, Rusch VW. Robotic assistance for video-assisted thoracic surgical lobectomy: technique and initial results. J Thorac Cardiovasc Surg 2006;131:54-9.

3. Stamenkovic SA, Agrawal DA, Clark SC, et al. Robotic Lobectomy using a Subxiphoid Approach. Available online: https://www.ctsnet.org/article/robotic-lobectomyusing-subxiphoid-approach

4. Cerfolio RJ, Bryant AS. How to teach robotic pulmonary resection. Semin Thorac Cardiovasc Surg 2013;25:76-82.

5. Craig SR, Walker WS. A proposed anatomical classification of the pulmonary fissures. J R Coll Surg Edinb 1997;42:233-4.

Cite this article as: Stamenkovic S, Melfi F. Right roboticassisted thoracoscopic lower lobectomy. Ann Cardiothorac Surg 2019;8(2):279-285. doi: 10.21037/acs.2019.03.04 